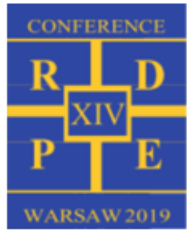

\title{
The cost-effectiveness of an energy storage system using hydrogen and underground gas storage
}

\author{
Sebastian Lepszy ${ }^{1}$ \\ ${ }^{1}$ Silesian University of Technology, ul. Konarskiego 18, 44-100 Gliwice, sebastian.lepszy@ polsl.pl, Poland
}

\begin{abstract}
Due to the random nature of the production, the use of renewable energy sources requires the use of technologies that allow adjustment of electricity production to demand. One of the ways that enable this task is the use of energy storage systems. The article focuses on the analysis of the cost-effectiveness of energy storage from the grid. In particular, the technology was evaluated using underground hydrogen storage generated in electrolysers. Economic analyzes use historical data from the Polish energy market. The obtained results illustrate, among other things, the proportions between the main technology modules selected optimally in technical and economic terms.
\end{abstract}

\section{Introduction}

Energy storage technologies make it possible to change the way electricity networks operate. Currently, the amount of energy produced and consumed is balanced in short periods, due to the low storage capacity. Increasing energy storage capacity can help in some cases reduce costs and pollutant emissions. Storage systems can also provide additional services for power networks such as: frequency regulation, rotating reserve, voltage regulation, reactive power compensation, network stabilization [1].

In Poland, there is little use of storage systems. The installed capacity of storage systems in the form of pumped storage power plants is $1413 \mathrm{MW}$, which represents $3.2 \%$ of the installed capacity of the power plant in Poland. The electricity produced in these systems represents only $0.27 \%$ of the electricity produced in Poland, and their capacity is about 10G Wh.

The main reason for considering energy storage should be making a profit for the energy storage company. This purpose of running a business also guarantees rational use of resources. Making a profit by the storage company means that it is possible to operate conventional power plants with greater power during periods of low demand for electricity and still with full power during periods of peak demand. The effect of such work may be an increase in the efficiency of electricity production of a conventional power plant, which may led to reduction in energy costs and a decrease in the production of electricity in peak systems.

Systems of this type can also contribute to reducing emissions by enabling greater use of overproduction of energy from renewable sources.

The current weak development of this technologies suggests that this are uneconomical solutions [2]. However, the rapidly changing economic environment should be taken into account, which is largely influenced by legal regulations, concerning the reduction of pollutant emissions and the use of renewable energy sources. The same regulations may also cause a high risk of introducing new technologies.

One of the significant factors affecting the economic efficiency of energy storage is the cost of energy transmission. In economic analyzes, for simplicity, transmission costs have been omitted, which corresponds to the situation of location of the storage system together with the power plant generating electricity.

The analyzes also are based on the assumption that the introduction of the storage system will not significantly affect price levels.

In the literature, it is difficult to find informations on optimal technology parameters in terms of power and storage capacity. The available analyzes mainly concern storage options in a short time. For example, in [3] an economic analysis of storage systems based on price arbitrage was conducted. The compared technologies did not contain, unfortunately, energy storage technology in the form of hydrogen. Economic profitability was demonstrated by CAES technologies and pumped storage power stations. All technologies had a storage capacity of less than one day, which made it impossible to take advantage of seasonal fluctuations in energy prices. In the literature, you can find descriptions of algorithms for assessing energy efficiency or economic energy storage systems cooperating with renewable energy sources. In [4] an algorithm for energy and economic calculations for a distributed system was presented, in which the energy store was located near a wind farm. Determined economic and energy parameters partly based on average values taking into account the normal distribution of probability. Although the results were obtained for the whole year, it was based on average prices of wind energy and production, which did not allow for the assessment of solutions in the case of long-term storage.

This article presents the methodology for selecting the size of machines and devices in the installation, and sets 
out the main economic parameters for estimating the profitability conditions of this type of installation

\section{Electricity prices}

To simplify the analysis, in order to estimate the economic effects, no additional costs of electricity transmission were assumed in further calculations.

The costs of purchasing energy can be determined by analysing prices on energy markets. Their analysis reveals the possibility of using price fluctuations to generate revenue in an energy storage company.

Figure 1 presents an ordered price chart on the Polish Power Exchange for the day-ahead market in 2017. The arithmetic average price is 158.37 PLN, the maximum to average ratio is 6.2 and the minimum to average ratio is 0.47 .

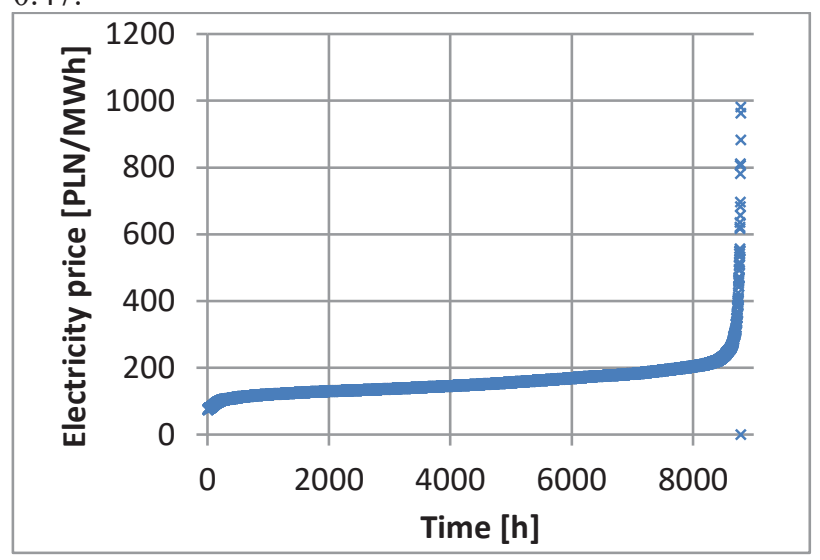

Fig. 1. A $n$ ordered chart of electricity prices (day-ahead market) on the Polish Power Exchange in 2017

Based on the data, the relationship between the average price of energy production and energy consumption for different levels of storage efficiency and the ratio of charging and generating power can be analyzed. The choice of the listed variables makes it easy to determine the difference in revenues and costs of electricity in the enterprise, e.g. to the amount of energy taken from the grid. For selected variables, revenues from the sale of electricity $S_{e l}$ can be specified:

$$
S_{e l}=C_{s r S} E_{S}
$$

where: $C_{s r}$ - average sale price of electricity for its production in the $\tau_{S}$ period characterized by the highest prices on the energy market; $E_{s}$ - the amount of energy sold.

Electricity purchase costs are calculated using following equation:

$$
K_{e l}=C_{s r K} E_{K}
$$

where: $C_{s r K}$ - average purchase price of electricity for its collection in the period $\tau_{K}$ characterized by the lowest prices on the energy market; $E_{k}$ - the amount of energy purchased.

There is a relationship between the amount of purchased and generated energy:

$$
E_{S}=\eta_{m} E_{K}
$$

where: $\eta_{m}$ - energy storage energy efficiency.

By specifying the ratio of storage loading power (power taken from the network) and storage discharge power (produced power, fed into the grid), it can be written:

$$
\tau_{S}=\eta_{m} \frac{N_{K}}{N_{S}} \tau_{K}
$$

where: $\tau_{\mathrm{s}}$ - storage discharge time with constant $\mathrm{N}_{\mathrm{S}}$ power; $\tau_{\mathrm{k}}$ - storage loading time with constant $\mathrm{N}_{\mathrm{K}}$ power. For analyses carried out for the year, it can be seen that the maximum time of energy consumption should be less than 8,760 hours, and the production time is its function. Using the data cited in Fig. 1, it is possible to determine the dependence of the price difference $C_{s r} S^{-} C_{s r k}$ as a function of energy consumption time $\tau_{\mathrm{k}}$, for different power ratios, for a given storage efficiency. Figures 2,3,4 show these functions for a storage efficiency of $0.2,0.5$ and 0.8 respectively.

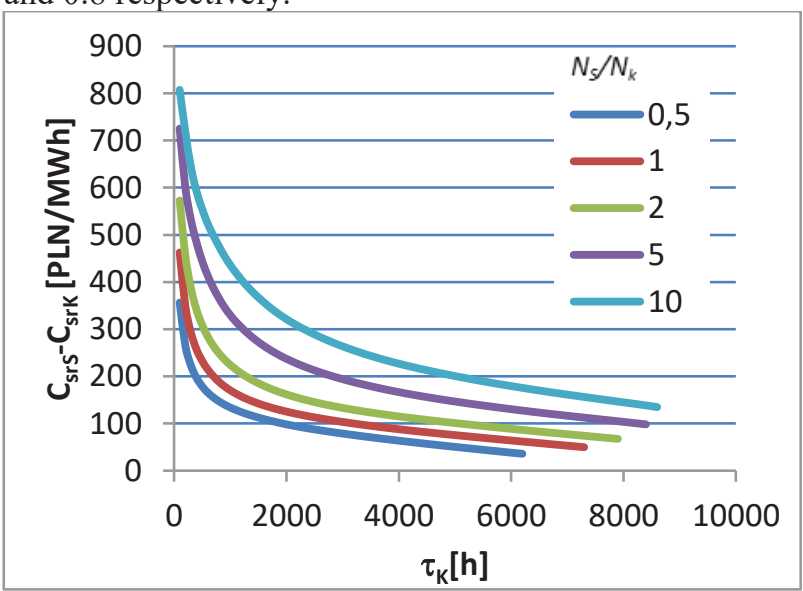

Fig. 2. Relationship between the average sale and purchase price difference $C_{s r S}-C_{s r k}$ for different value of working time $\tau_{k}$ and the ratio of production and consumption power $N_{S} / N_{k}$ for storage efficiency $\eta_{m}=0.2$

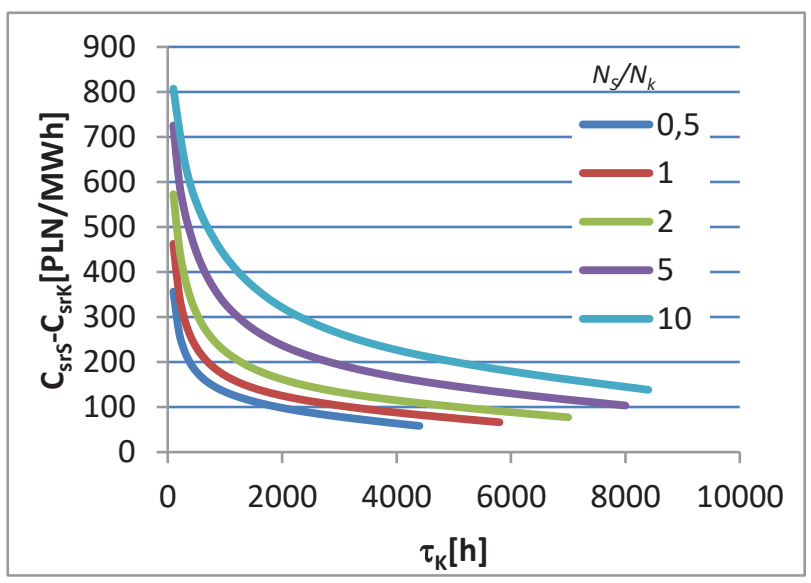

Fig. 3. Relationship between the average sale and purchase price difference $C_{s r s}-C_{s r k}$ for different value of working time $\tau_{k}$ and the ratio of production and consumption power $N_{S} / N_{k}$ for storage efficiency $\eta_{m}=0.5$ 


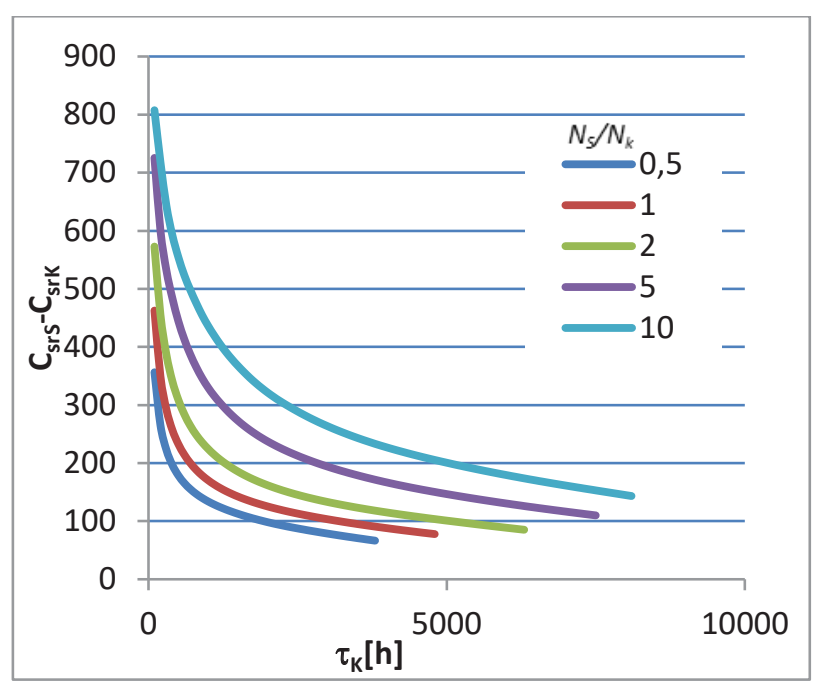

Fig. 4. Relationship between the average sale and purchase price difference $C_{s r s}-C_{s r k}$ for different value of working time $\tau_{k}$ and the ratio of production and consumption power $N_{S} / N_{k}$ for storage efficiency $\eta_{m}=0.8$

Based on the analysis, it can be concluded that the highest prices differences can be obtained only for a very short time of energy consumption, and thus for the production of energy from storage in periods of highest prices. Increasing the ratio of generation capacity to consumption capacity increases the difference in average prices due to the possibility of producing energy from the storage with greater power during high prices.

Increasing storage efficiency reduces price differences for individual values of energy consumption times, due to the extension of the time of energy production from the storage at lower prices.

\section{Electricity costs and revenues}

The price difference, although it is a factor enabling economic efficiency, does not directly illustrate the scale of the project. For small amounts of time consuming electricity, the price difference is large, while the amount of stored energy is small, which results in a small difference in revenues and costs of electricity. On the other hand, for a large time of energy consumption, the production time is also long, while the price difference is small, which means that the difference in revenues and costs will also be small. To illustrate this issue, based on the data, the dependence of the difference in revenues and costs was developed for installations with $1 \mathrm{MW}$ input power for different values of $N_{S} / N_{k}$ power and storage efficiency (Fig. 5, 6, 7). For a relatively low storage efficiency, it can be determined that only for a relatively short storage operation time and a large $N_{S} / N_{k}$ power ratio the difference in revenues and costs is positive. The main reason is the low value of storage efficiency. The increase in storage efficiency increases the value of the difference in revenues and costs as well as the optimal loading time of the storage.

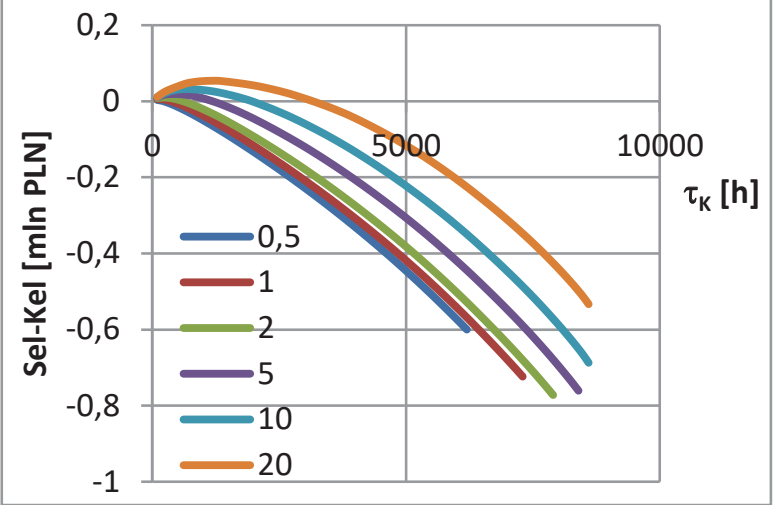

Fig. 5. D ependence of the difference in revenues and costs of electricity $S_{e l}-K_{e l}$ for different values of working time $\tau_{k}$ and the ratio of production and consumption power $N_{S} / N_{k}$ for storage efficiency $\eta_{m}=0.2$

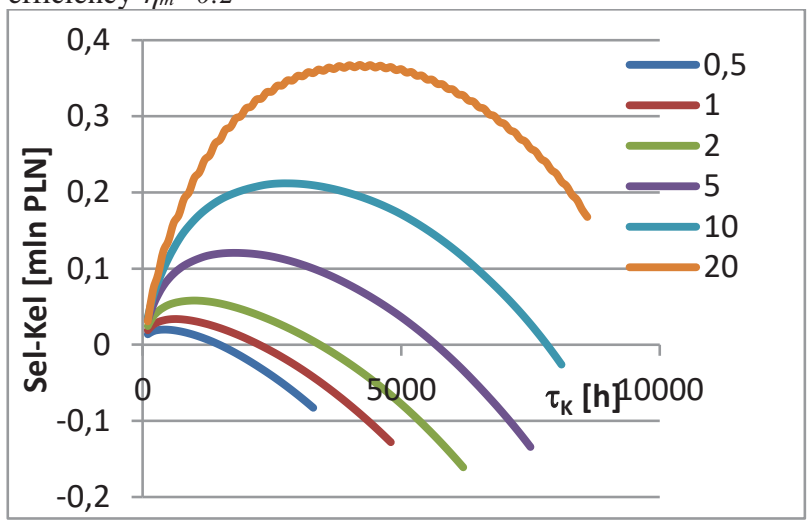

Fig. 6. Dependence of the difference in revenues and costs of electricity $S_{e l-} K_{e l}$ for different values of working time $\tau_{k}$ and the ratio of production and consumption power $N_{S} / N_{k}$ for storage efficiency $\eta_{m}=0.5$

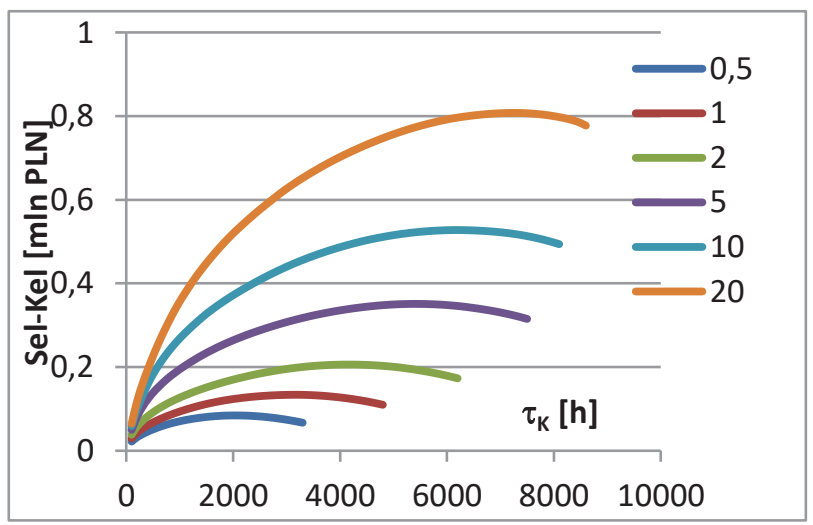

Fig. 7. Dependence of the difference in revenues and costs of electricity $S_{e l}-K_{e l}$ for different values of working time $\tau_{k}$ and the ratio of production and consumption power $N_{s} / N_{k}$ for storage efficiency $\eta_{m}=0.8$

\section{Relative profit}

Increasing the difference in revenues and costs for large power ratios tends to analyze the ratio between the said difference and the sum of the system power in the storage loading and unloading mode. This analysis is interesting because the sum of power is a parameter that significantly affects the investment expenditure related to the construction of the system. Figures $8,9,10$ 
illustrate these relationships for different storage efficiency values. The highest revenue values in relation to the total power can be obtained for the highest storage efficiency. Presented charts, also take into account the impact of the power ratio and working time on the optimal size of the storage and related investment outlays. The presented analysis can lead to rational conclusions when investment outlays for gas storage would be proportional to the sum of charging and discharging power.

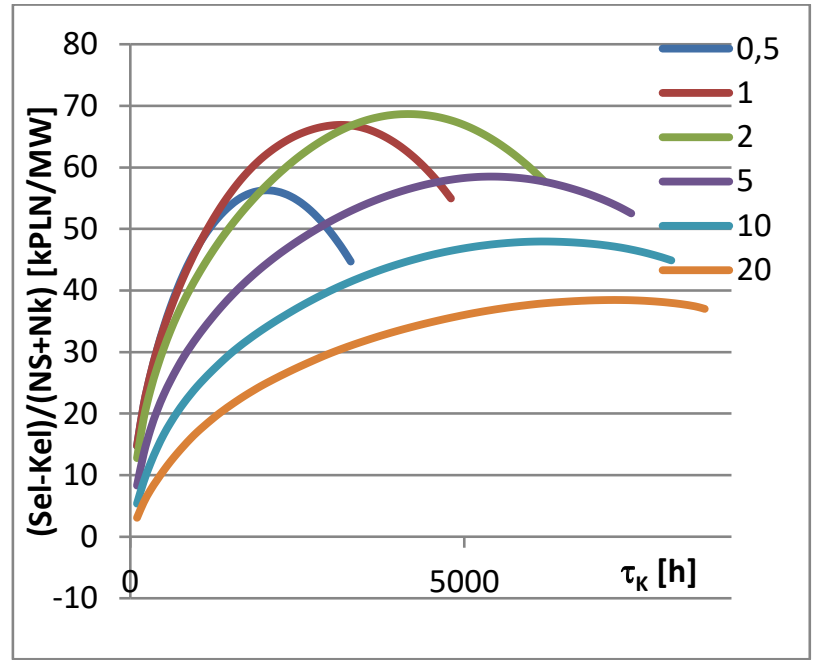

Fig. 8. Dependence of the profit to power ratio for different values of working time $\tau_{k}$ and the ratio of production and consumption power $N_{s} / N_{k}$ for storage efficiency $\eta_{m}=0.8$

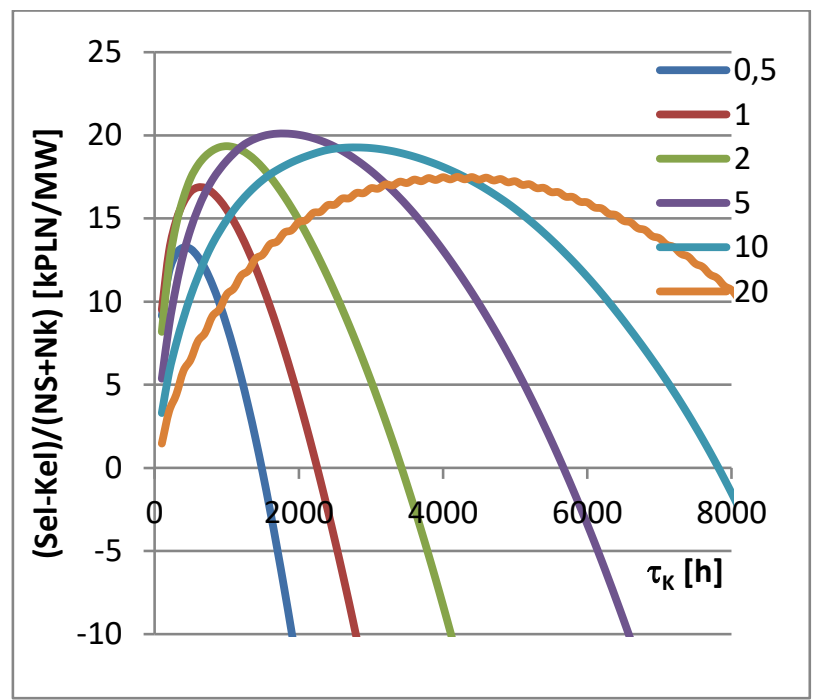

Fig. 9. Dependence of the profit to power ratio ratio for different values of working time $\tau_{k}$ and the ratio of production and consumption power $N_{S} / N_{k}$ for storage efficiency $\eta_{m}=0.5$

The presented methodology illustrates the possibilities of obtaining revenues for energy storage installations assuming no transmission costs. Including them further reduces the price difference, which can significantly affect the company's energy efficiency.

The presented analysis allows only to estimate the basic parameters of the storage system operating based on one selected market and based on the price level of the selected time. Significant changes in the energy market that we observe nowadays can cause real changes in the results of analyzes.

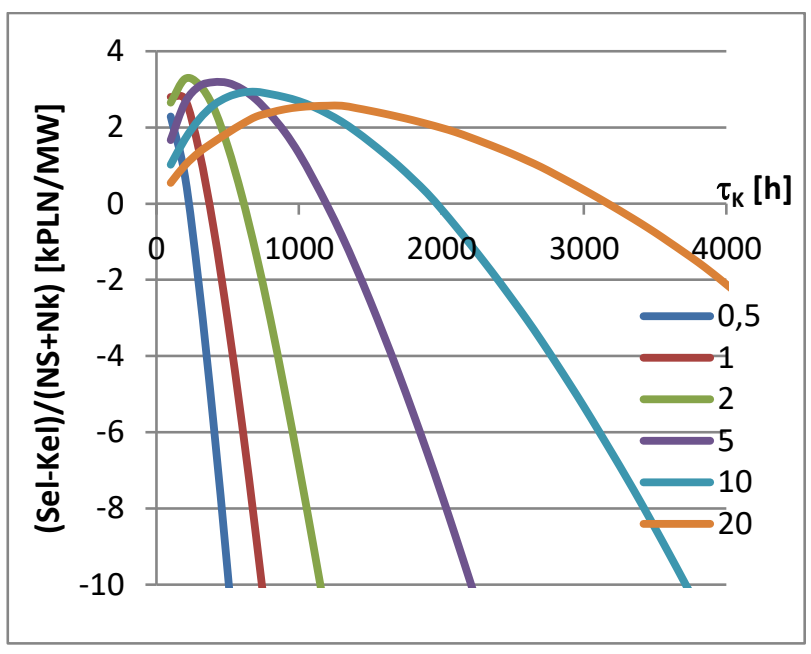

Fig. 10. Dependence of the profit to power ratio for different values of working time $\tau_{k}$ and the ratio of production and consumption power $N_{S} / N_{k}$ for storage efficiency $\eta_{m}=0.2$

\section{Gas storage capacity}

To conduct a preliminary economic analysis of the energy storage system, in addition to information about the energy consumption of energy production and, therefore, the power of devices, information on the size of the energy storage is necessary, which, inter alia, affects investment expenditure related to the construction and operating costs of the system.

Based on the time course of energy prices in a given market, the time intervals in which energy is stored or taken from the storage can be specified. Assuming constant energy flows, it is possible to specify the time variability of the amount of gas in the storage system. Assuming the value of gas losses from the storage system, it is possible to determine its working capacities as the difference from the maximum and minimum amount of medium in the cavern

The analysis of the energy storage capacity value can be carried out based on such parameters as:

- ratio of gas charging and discharging flow;

- time of charging the storage in a year.

For each time interval it is possible to determine if the price that can be obtained when buying is less than the limit price, which is related to the time the system works in the loading process. If the above inequality occurs, we assume that in this time interval the storage is loaded with the assumed capacity.

Similarly, for each time interval, it is possible to determine, if the energy price is greater than the cut-off price, related with the system operation time in the unloading process. If this inequality occurs, the gas is unloaded with capacity depending on the assumed flow ratio. There may be periods of time for which the gas storage is not unloaded or loaded. Also in the long term (e.g. year), the quantity introduced into the storage must 
be balanced with the amount of gas released. On this basis, it can be written:

$$
\tau_{S}=\left(1-s_{m}\right) \frac{V_{K}}{V_{S}} \tau_{K}
$$

where: $V_{k}$ - gas storage loading flow; Vs - gas storage discharge flow; $s_{m}$ - relative gas losses related to charging efficiency.

An example of the dependence of the amount of gas in the storage over time is illustrated in Fig. 11. It was assumed that the capacity ratio is equal to 4 and the loading time is $7000 \mathrm{~h}$. The amount of stored gas is expressed using the $E_{m \_} h$ value:

$$
E_{m_{-} h}=\sum_{t=1}^{8760} V(t) \Delta t
$$

where: $V(t)$ - time function of charging flow (in mass or mol units charging flow per h); $\Delta t$ - time interval (1h). From the chart you can read that for a selected year of operation the gas storage should be emptied in about 4000 hour of operation from the beginning of the year and filled in about 1000 hour of work per year, respectively.



Fig. 11. Dependence of the amount of stored gas in time for working time $\tau_{k}=7000 \mathrm{~h}$ and the ratio of gas production and consumption flow $V_{k} / V_{s}=4$

The dependence of the required storage capacity can be determined as a function of the running time of the energy consuming system. The results for different values of storage loading and unloading efficiency are shown in Fig. 12. It can be seen that for low values of the charging time the storage capacity is almost linearly dependent on the said time. This is directly related to high energy prices far exceeding the average value and the need to store this part of energy until the low price. For larger values of the charging time, changes in capacity are no longer always increasing.

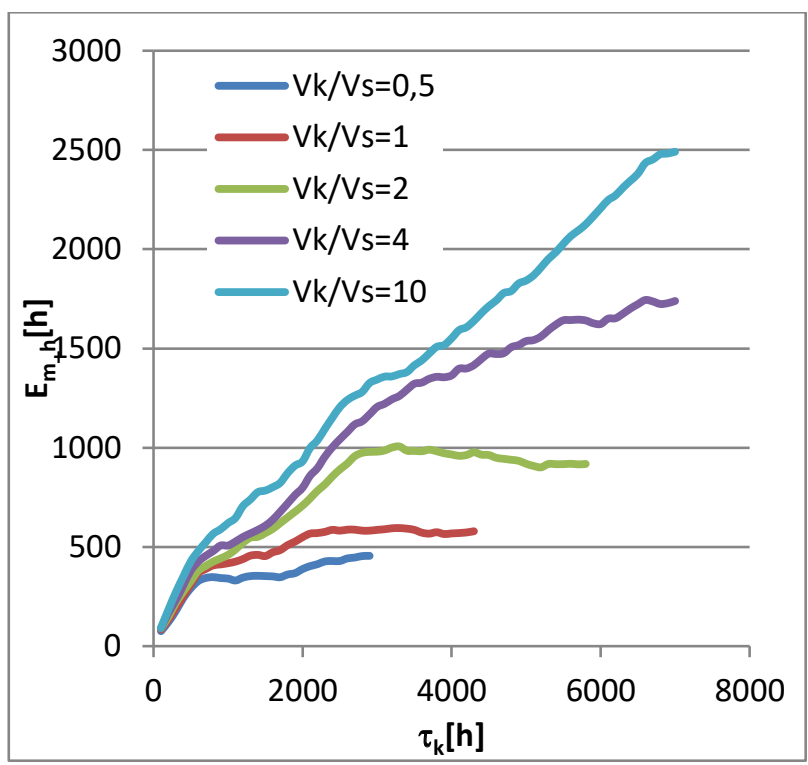

Fig. 12. Dependence of the necessary working capacity in ours of gas production as a function of working time $\tau_{k}=$ and the ratio of gas production and consumption flow $V_{k} / V_{s}=4$

For the example, installation with the $V_{k} / V_{s}$ ratio of 4 and loading time of the storage 1000 we get 277 beginnings of the charging system operation and a similar number of 273 beginnings of the discharge system operation. The maximum continuous operation time of the charging system is 168 hours, and for the discharge - 16 hours.

\section{Storage of energy using hydrogen - case study}

The profitability analysis of storage theology was conducted based on the system block diagram presented in Fig. 13.

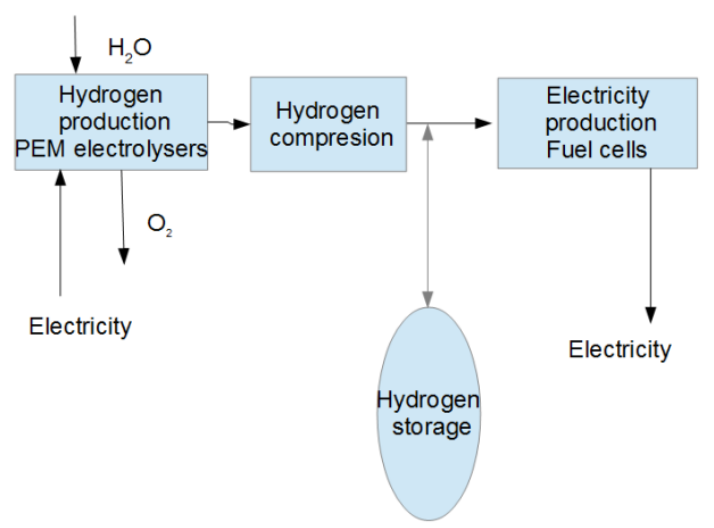

Fig. 13. B lock diagram of system

In the presented system, electricity is converted into chemical energy of hydrogen in PEM type cells. Unlike alkaline electrolysers, these devices have a better development perspective, in particular in terms of electricity conversion efficiency or capital expenditure. It was assumed that their energy efficiency related to LHV is at the level of $68 \%$, which is the value possible to achieve in the near future [5]. 
Hydrogen from the cells is directed to the compressor system increasing the pressure to the level required in the gas storage. To simplify the analysis, electricity consumption in this module was omitted. This consumption can be from $0 \%$ (pressure in the gas storage below 30 bar) to $2 \%$ (pressure in the gas storage approx. 200bar) of energy consumed by electrolysers.

The compressed hydrogen goes to the gas storage in the form of e.g. salt caverns

Production of electricity takes place in PEM type fuel cells with 58\% energy conversion efficiency (LHV based).

These values allow to determine the maximum storage efficiency at the level of $39.4 \%$. It was assumed that the ratio of electricity production power to input power is 4.7, which is the optimal value for charging time of 1100 hours a year.

Assuming the power of the energy intake system at $50 \mathrm{MW}$, it is necessary to use fuel cell modules with 235MW electric power. These power values correspond to $1.02 \mathrm{Mg} / \mathrm{h}$ hydrogen production and $12.15 \mathrm{Mg} / \mathrm{h}$ utilization rate. Based on the performance ratio, the gas storage working capacity of $673 \mathrm{Mg}$ can be estimated.

Based on the day-ahead market data of the 2017 energy exchange, it can be determined that the cost of purchasing electricity would be 6022 000PLN while revenues from the sale of energy $9730000 \mathrm{PLN}$, which gives a difference of 3707 000PLN. The difference obtained can be compared to capital expenditure on main devices, i.e. electrolysers and fuel cells. Assuming the unit price of electrolysers at the level of 1000 Euro/kWe and fuel cells at the level of 3000 Euro/kWe, we obtain capital expenditure worth $216000000+3046000000$ $=3262$ million PLN, which is about 900 times greater than the difference calculated earlier

\section{Conclusions}

The day-ahead market of the commodity energy exchange was characterized in 2017. a small number of hours in which the price was much lower than the average and a small number of hours with the price significantly exceeding the average price. This price distribution theoretically enables the operation of the storage system using price arbitrage, with particular emphasis on the operation of the system during high prices.

Taking into account energy balancing in the storage system, it is possible to determine the difference in purchase costs and revenues from the sale of electricity. The highest values of this indicator can be obtained for a relatively high power of the electric energy production system in relation to the power of the electric energy consuming system. The increase in the ratio of the production system's power to the consumed power caused the increase of the optimal value of the time of energy consumption.

The day-ahead market of the commodity energy exchange was characterized in 2017 by a very small number of hours in which the price was much lower than the average and a small number of hours with the price significantly exceeding the average price. This price distribution theoretically enables the operation of the storage system using price arbitrage, with particular emphasis on the operation of the system during high prices.

Taking into account energy balancing in the storage system, it is possible to determine the difference in purchase costs and revenues from the sale of electricity. The highest values of this indicator can be obtained for a relatively high power of the electric energy production system in relation to the power of the electric energy consuming system. The increase in the ratio of the production system's power to the consumed power caused the increase of the optimal value of the time of energy consumption.

Due to significant changes in the value of revenues from the purchase and sale of electricity depending on the power ratio, the ratio of revenues in relation to the power of main elements was determined. Optimal working times of the collection system were on the same level like optimal values taking into account only revenues.

A case study for a system using PEM electrolysers and fuel cells indicates the lack of economic efficiency of the considered solution for the market in 2017.

\section{References}

[1] M. Arbabzadeh, J.X. Johnson, G.A. Keoleian: Parameters driving environmental performance of energy storage systems across grid applications. $J$ Energy Storage, 12 (2017), pp. 11-28

[2] E. Fertig, J. Apt: Economics of compressed air energy storage to integrate wind power: a case study in ERCOT. Energy Policy, 39 (5) (May 2011), pp. 233023422

[3] Bradbury K., Pratson L., Patińo-Echeverri D.: Economic viability of energy storage systems based on price arbitrage potential in real-time U.S. electricity markets. Applied Energy 114 (2014) 512-519

[4] M. Korpaas, A. T. Holen, R. Hildrum, Operation and sizing of energy storage for wind power plants in a market system, International Journal of Electrical Power \& Energy Systems 25 (8) (2003) 599-606.

[5] Bertuccioli L., Chan A., Hart D., Lehner F., Madden B., Standen E.: Study on development of water electrolysis in the EU. Fuel Cells and Hydrogen Joint Undertaking, Final Report; February 2014 